\title{
Panoramic Movie Generation Using an Omnidirectional Multi-camera System for Telepresence
}

\author{
Sei Ikeda, Tomokazu Sato, and Naokazu Yokoya \\ Graduate Scho ol oflnformation Science, Nara Institute of Science and T echnology \\ 8916-5 T ak ayamalkoma, 630-0101 Nara, Japan \\ \{sei-i, tomoka-s, yokoył@is.aist-nara.ac.jp \\ http://yokoya.aist-nara.ac.jp/ ${ }^{\sim}$ sei-i/research/
}

\begin{abstract}
T elepresencesystems using an omnidirectional image sensor enable us to exp erience remote sites with rich sensation. An omnidirectional $\mathrm{m}$ ulti-camerasystem is more useful to acquire high-resolution omnidirectional images of outdoor scenes than a monocular camera system. Ho w ever, exactalibration of the camera system is necessary to generate a panoramic movie. In this pap er, w e describ e panoramic movie generation method based on geometric and photometric calibration of the omnidirectional m ulti-camerasystem. Some protot yp esof telepresence system are also shown as applications of generated panoramic movies. These high-resolution telepresence systems ha ve been proøn to enable us to $\exp$ erienceremote sites with high presence.
\end{abstract}

\section{Introduction}

T elepresencesystems using an omnidirectional image sensor enable us to exp erience remote sites with rich sensation. These systems are exp ectedto be usedin the fields of education and en tertainment. A curv edmirror [1] and a fish eye len s [2] have beenused for these telepresence systems b ecause they can be attac hed to a standard camera. How ev er,such methods ha vesome problems concerning the shortage and p osition-dependency of image resolution, b ecause these sensors capture an omnidirectional scene by a single camera. Though a higher resolution telepresence system is also implemented by using an HD camera and a curv edmirror [3], it do es notsolv e theproblem of p osition-dependency of image resolution.

On the other hand, an omnidirectional multi-camera system can obtain a high-resolution image with almost uniform resolution. How ev er,exact camera calibration is necessary to generate a panoramic image from multiple images acquired by such asystem.

In this paper, w epropose a camera calibration and panoramic movie generation method for the omnidirectional multi-camera system "Ladybug". In exp eriments, Ladybug is calibrated and a panoramic movie is generated based on the proposed methods. As applications of the generated panoramic movie, three protot yp esf telepresence system are also demonstrated. 


\section{Camera Calibration for Omnidirectional Multi-camera System}

This section describes a method of geometric and photometric calibration for an omnidirectional multi-camera system[4]. As an omnidirectional multi-camera system, we use Ladybug made by Point Grey Research Inc. shown in Figure 1. The camera unit consists of six cameras (Figure 1 (left)): Five radially configured on a horizontal ring and one pointing vertically. Figure 1 (right) shows a storage unit, which consists of four hard disks. The camera system can collect synchronized movies at 15 fps covering more than $75 \%$ of the full spherical view with almost the same apparent point of view.

\subsection{Geometric Calibration}

In the geometric calibration, intrinsic parameters and extrinsic parameters of each camera should be estimated. The extrinsic parameters are estimated in a unified coordinate system to obtain the relationship among six cameras. In order to estimate camera parameters accurately, spatial arrangement of many markers of known 3D position is required.

First, arrangement of markers are described. In our method, grid points of a lattice pattern on a calibration board are used as markers. 3D coordinates of four corners of the calibration board are measured by a totalstation and all $3 \mathrm{D}$ positions of the markers on the board are calculated by linear interpolation among its corners. The calibration board is arranged and measured at several different positions for acquiring a large number of makers' $3 \mathrm{D}$ positions and $2 \mathrm{D}$ positions on images as shown in Figure 2. In our method, the totalstation must be fixed on the same position while the whole geometric calibration procedure is performed. Consequently, 3D positions of all markers captured by all the cameras can be represented in a single coordinate system made by the totalstation.

Next, the camera parameters of each camera are estimated. The intrinsic parameters (focal length, lens distortion parameters, center of distortion, aspect ratio) are estimated by using the Tsai's method[5]. Note that the distortion parameter $\kappa_{2}$ in Tsai's method is also considered because input images from the cameras are highly distorted.

In this paper, extrinsic parameters (position, orientation) of each camera $c(c=1,2, \ldots, 6)$ are estimated by minimizing the sum of re-projection error $E_{c}=\sum_{m}\left(\boldsymbol{u}_{\boldsymbol{m}}-\boldsymbol{v}_{\boldsymbol{m}}\right)^{2}[6]$, which is the sum of squared distances on the image between the projected position $\boldsymbol{v}_{\boldsymbol{m}}$ of measured $3 \mathrm{D}$ position and the detected $2 \mathrm{D}$ position $\boldsymbol{u}_{\boldsymbol{m}}$ of the marker $m$.

\subsection{Photometric Calibration}

In the photometric calibration, the limb darkening and color balances are corrected. First, the correction of the limb darkening is described. The limb darkening is a gradually decreasing effect of brightness in peripheral regions in images 


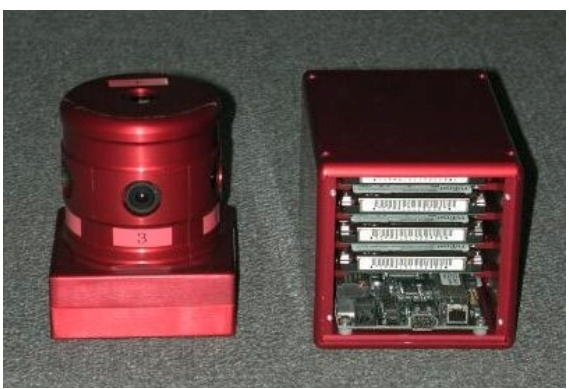

Fig. 1. Camera head (left) and storage unit (right) of Ladybug

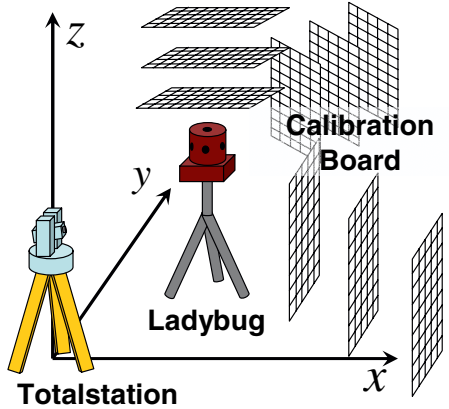

Fig. 2. Arrangement of calibration board in space

acquired by a wide-angle camera. It is known that the $\cos ^{4} \theta$ phenomenon is proper to a wide-angle lens. Thus we treat the $\cos ^{4} \theta$ phenomenon and correct image intensities using the estimated focal length and a lens diameter of a design parameter of a camera [7].

Next, color balances among cameras are corrected by capturing the same scene by all the cameras. Generally it is known that the irradiance of the image and the radiance of the surface have a linear relation. Therefore, the color balances between the cameras are linearly adjusted. The linear transformation parameters of colors are determined by equalizing the RGB histograms of all the images.

\section{Generating Omnidirectional Panoramic Movies}

In generation of a panoramic image, the limb darkening and the color balance of input images are corrected in advance. Then, corrected images are projected on a spherical or cylindrical projection surface by using the intrinsic and extrinsic parameters obtained by the geometric calibration. In this section we describe a projection method of the corrected images.

The method is based on re-projecting calibrated input images on a spherical or cylindrical virtual image surface. Since the centers of projection of multiple cameras of the omnidirectional multi-camera system are different from each other, the single viewpoint perspective projection model is not applicable for this system. However, when the distance of a target from the system is sufficiently large, the centers of projection can be considered as the same. Therefore, we assume that the target scene is far enough from the system and set the spherical or cylindrical projection surface $S$ far enough from the camera system. The center of the projection surface $S$ is set at the center of gravity of all the camera's center of projection. A panoramic image is generated by projecting all the pixels of all the images onto the projection surface $S$. Note that a blending technique is 
used for generating a smooth panoramic image, when a point on the projection surface $S$ is projected from multiple images of different cameras.

\section{Experiments}

We have calibrated the Ladybug system and have generated a panoramic movie. This section describes some experimental results concerning geometric and photometric calibration and panoramic movie generation. Three prototypes of telepresence system are also developed as applications of high-resolution panoramic movies.

\subsection{Generation of Panoramic movies}

\section{(1) Calibration of Ladybug}

In the geometric calibration, 3D positions of grid points on the calibration board were measured by a totalstation LEICA TCR1105 XR. The 2D positions on input images are detected at sub-pixel resolution from the calibration board images captured by each camera of Ladybug fixed on a tripod. The calibration board was arranged and captured at different depth from the fixed camera system three times. Consequently, more than 500 pairs of 3D and 2D positions of grid points were obtained and used for calibration for each camera.

Figure 3 shows the result of reducing geometric distortion of an input image using the estimated intrinsic parameters. We can confirm that the intrinsic parameters are fairly good. Figure 4 shows the result of correcting the limb darkening. The left is an input image. The right is a corrected image for the shortage of brightness in peripheral regions of the input image. Figure 5 shows a part of panoramic images before and after the color balance adjusting. In the right image, the boundary lines do not stand out compared with the non-corrected image in the left.

\section{(2) Generation of a Panoramic Movie}

A panoramic movie was generated by using the result of calibration described in the previous section. The input movie was obtained by setting Ladybug put on a moving car. Figure 6 shows an example set of input images (resolution: $768 \times 1024$ each). A panoramic image (resolution: $3840 \times 1920)$ generated from these input images is also shown in Figure 7. This panoramic image is unfolded in polar coordinates from the spherical coordinate representation. A black part at the bottom of the panoramic image is the part of no input images. Since any boundary lines between different camera images are hardly found in Figure 7, we can confirm that the geometrical and photometrical calibration is successfully achieved. We can not perceive any boundary lines in the panoramic image except objects very close to the camera system. 


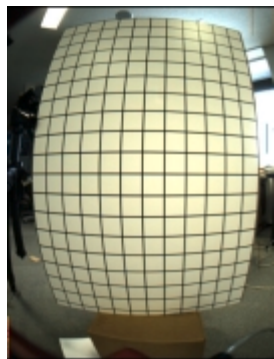

(a) input

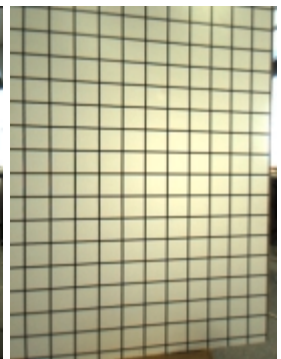

(b) corrected

Fig. 3. Lens distortion correction

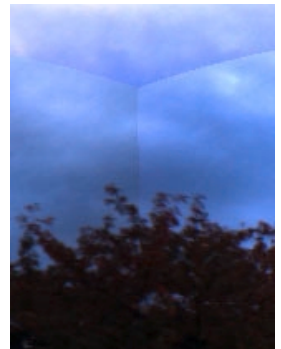

(a) non-corrected

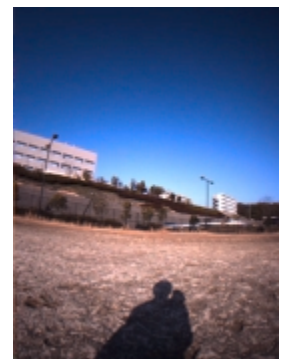

(a) input

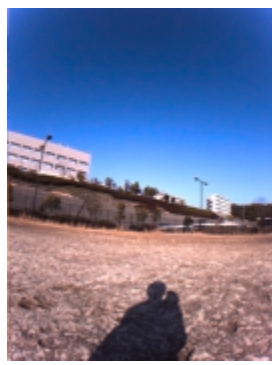

(b) corrected

Fig. 4. limb darkening correction

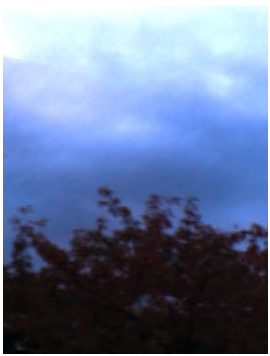

(b) corrected

Fig. 5. Color adjustment

\subsection{Prototype Telepresence Systems}

This section briefly describes three prototype telepresence systems developed as applications of high-resolution panoramic movies. Three telepresence systems are equipped with different immersive displays as shown in Figure 8: (a) a spherical display (Elumens VisionStation), (b) a face mounted display (Olympus EyeTrek) and (c) cylindrical display (CYLINDRA), which provide users different usage and interaction. Our systems enable users to interactively view an omnidirectional scene by projecting a specified part of a panoramic movie in real time.

(a) Spherical display: A user can observe almost the half of omnidirectional panoramic view in the display as shown in Figure 8 (a). The direction of the view field can be controlled by a game controller (Microsoft SideWinder Game Pad Pro).

(b) Face mounted display: A user can view an omnidirectional view field naturally by turning his head as shown in Figure 8 (b). The direction of user's head is acquired by a magnetic sensor (Polhemus Fastrack): a source located on a table and a receiver attached on the face mounted display. 


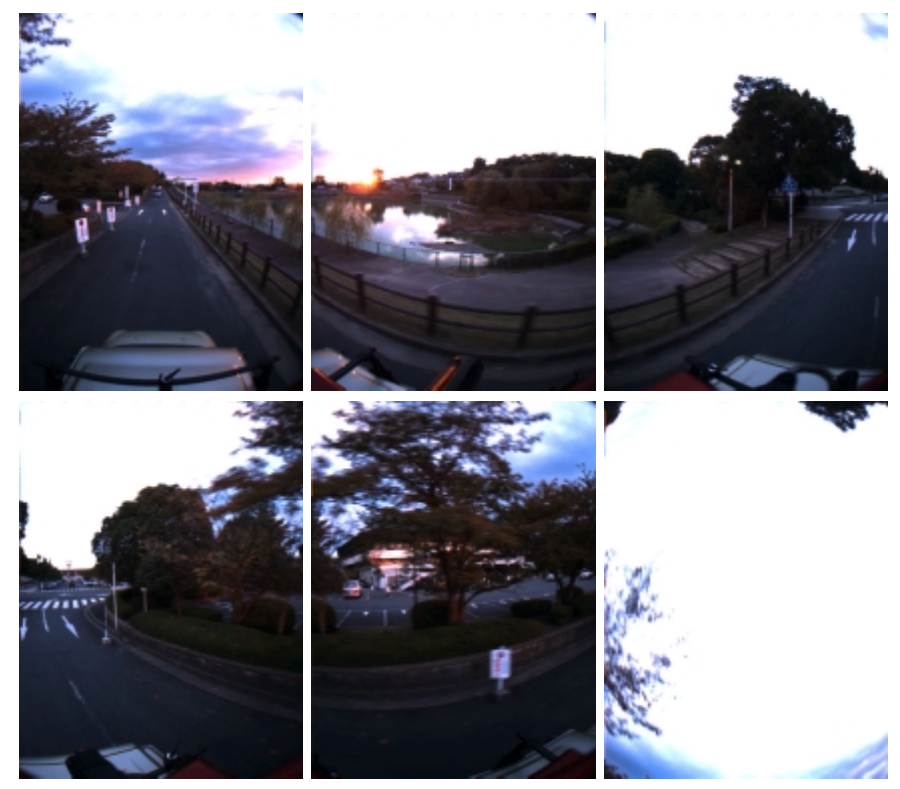

Fig. 6. Input images: upper camera (right end) and horizontal cameras (others)

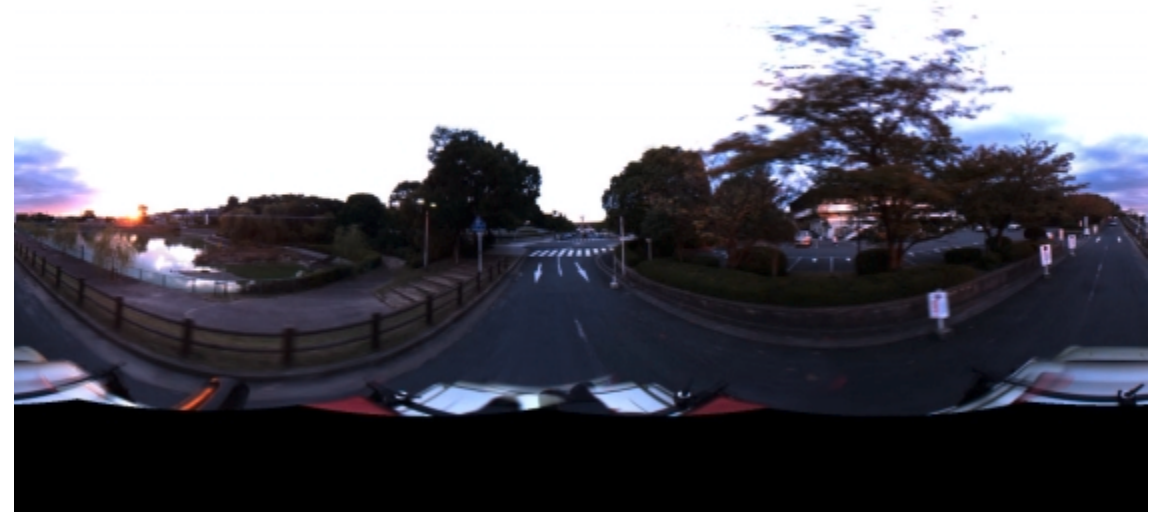

Fig. 7. One frame of spherical panoramic movie 
(c) Cylindrical display: In this system, the full horizontal range of omnidirectional movie is projected to a large cylindrical screen by six projectors. Multiple users can simultaneously view different directions as shown in Figure $8(\mathrm{c})$.

The frame rate of displaying images is $15 \mathrm{fps}$ on all the systems, which is the same as the image capt uring rate by Ladybug. We have confirmed that our telepresence systems exhibit high-presence of a remote site in respect of resolution, field of view, and interactive viewing.

\section{Conclusion}

In this paper, we have described a method for generating a high-resolution spherical panoramic movie based on the result of geometric and photometric calibration for an omnidirectional multi-camera system Ladybug. In the geometric calibration, a large number of markers were virtually arranged around the system by using a calibration board and a totalstation for improving the accuracy of calibration. We have also prototyped three telepresence systems which provide users with an omnidirectional field of view. Experiments have exhibited that telepresence systems using high-resolution panoramic movies provide us with the feeling of high presence in remote sites. In future work, a better lens distortion model for wide-angle lens will be investigated for obtaining higher accuracy in generation of panoramic movies.

\section{References}

1. Y. Onoe, K. Yamazawa, H. Takemura, N. Yokoya: Telepresence by Real-time Viewdependent Image Generation from Omnidirectional Video Streams. Computer Vision and Image Understanding 71(2):154-165, 1998

2. K. Miyamoto: Fish Eye Lens. Journal of Optical Society of America 54(8):10601061, 1964

3. K. Yamazawa, H. Takemura, N. Yokoya: Telepresence system with an Omnidirectional HD Camera. Proc. 5th Asian Conf. on Computer Vision (ACCV2002) 2:535-538, 2002

4. S. Ikeda, T. Sato, N. Yokoya: A Calibration Method for an Omnidirectional Multicamera System. Proc. SPIE Electronic Imaging 5006, 2003

5. R. Y. Tsai: A Versatile Camera Calibration Technique for High-accuracy 3D Machine Vision Metrology Using Off-the-shelf TV Cameras and Lenses. IEEE Journal of Robotics and Automation RA-3(4):323-344, 1987

6. T. Sato, M. Kanbara, N. Yokoya, H. Takemura: Dense 3D Reconstruction of an Outdoor Scene by Hundreds-Baseline Stereo Using a Hand-Held Video Camera. Int. Journal of Computer Vision 47(1-3):110-129, 2002

7. B. K. P. Horn. In: Robot Vision. MIT Press :206-209, 1986 


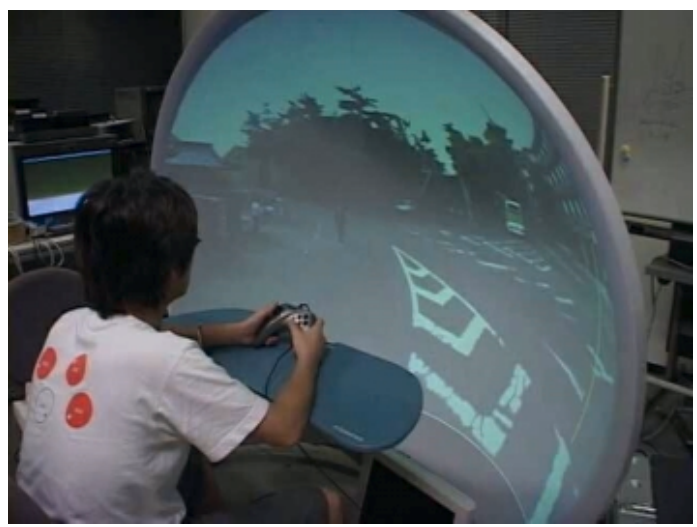

(a) spherical display

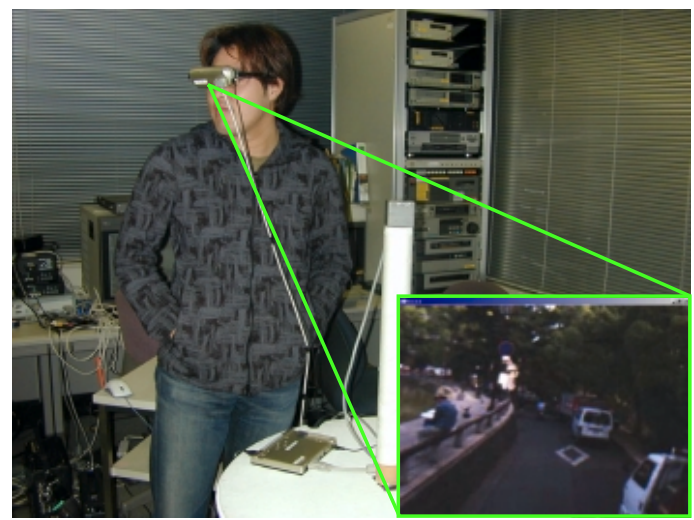

(b) face mounted display

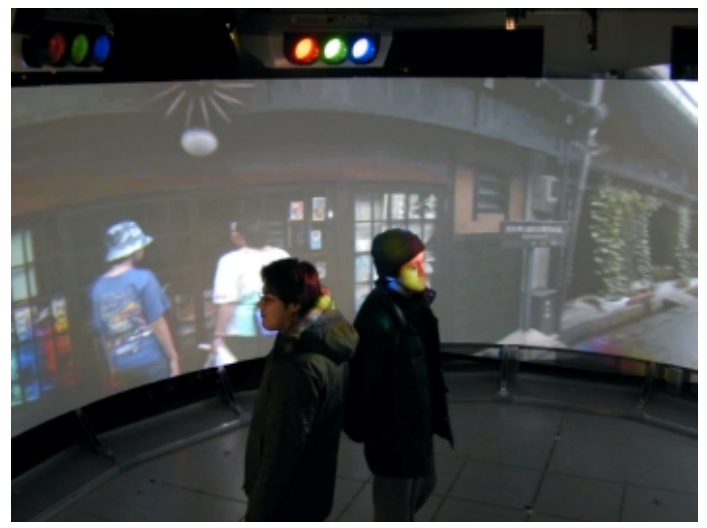

(c) cylindrical display

Fig. 8. Appearance of telepresence systems 